\title{
Negative emotional biases in late chronotypes
}

Charlotte Mary Horne ${ }^{1}$, Sara Daphne Margaret Marr-Phillips, Rabia Jawaid, Edward Leigh Gibson \& Ray Norbury

Ms Sara Daphne Margaret Marr-Phillips - marrphis@ roehampton.ac.uk

Ms Rabia Jawaid - jawaidr@ roehampton.ac.uk

Dr Edward Leigh Gibson - L.Gibson@ roehampton.ac.uk

Dr Ray Norbury - $\underline{\text { Ray.Norbury@ roehampton.ac.uk }}$

Addresses for co-authors:

Department of Psychology

University of Roehampton,

London, UK

${ }^{1}$ Address for correspondence:

Ms Charlotte Mary Horne

Department of Psychology

University of Roehampton

Whitelands College

London

SW15 4JD

E: hornec1@roehampton.ac.uk 


\begin{abstract}
Increasing evidence suggests evening chronotypes are at increased risk for developing depression. Here, we examined if, similar to acutely depressed patients, evening chronotype individuals display biases in emotional face recognition. Two hundred and twenty six individuals completed an online survey including measures of sleep quality, depression/anxiety and chronotype followed by a simple emotion recognition task presenting male and female faces morphed in 10 steps between $0 \%$ (neutral) and 100\% sad or happy. Evening chronotype was associated with increased recognition of sad facial expressions independently of sleep quality, mood, age and gender. The current results extend previous work indicating that negative biases in emotional processing are present in evening chronotypes and may have important implications for the prevention and treatment of depression in these vulnerable individuals.
\end{abstract}

Keywords: Chronotype; Depression; Negative bias; Cognition; Emotion; Facial expression 


\section{Introduction}

Differences in diurnal rhythmicity between individuals is referred to as morningnesseveningness (Adan and Almirall, 1991). These differences can be measured along a scale although they are commonly organised into three broad circadian groups (chronotypes): morning-types ('larks') who prefer to go to bed and rise early, evening-types ('owls') who prefer to go to bed and rise late, and intermediate chronotypes that fall in between.

Increasing evidence suggests that differences in circadian rhythmicity affects cognition and may also be involved in the aetiology and pathophysiology of depression. For example, late chronotypes are significantly more likely to report depressed mood, anhedonia, severe depressive symptoms, higher negative emotionality, a diagnosis of depression and are more likely to report use of prescribed antidepressant medication (Hidalgo et al., 2009, Merikanto et al., 2013, Merikanto et al., 2015, Simor et al., 2015).

Although the above converging findings indicate an association between late chronotype and depression, they do not address the underlying mechanisms that explain why late chronotypes are more at risk of developing the disorder. In an attempt to address this question, Berdynaj and colleagues (Berdynaj et al., 2016) explored emotional and attentional biases in never-depressed late (LC) and early/intermediate chronotypes (EIC). The authors found that LC individuals recognised a greater number of sad facial expressions and were slower at recognising negative vs. positive personality trait words compared to EIC individuals (Berdynaj et al., 2016). Additionally, EIC recalled significantly more positive personality trait words and displayed an attentional bias towards positive (happy) facial expressions. This pattern of results is similar to that seen in acutely depressed patients (Bradley et al., 1995, Peckham et al., 2010), remitted depressed patients (Anderson et al., 2011) and other groups at increased risk for depression [e.g. highly neurotic individuals 
(Chan et al., 2007)] and are consistent with cognitive theories of depression which suggest negative emotional bias is important in the aetiology and maintenance of depression (Beck, 1989).

A significant limitation, however, of the above study (Berdynaj et al., 2016) was the collapsing of morning- and neither-type participants into a single group. The current study, therefore, aims to extend these earlier findings by exploring expression recognition in a large sample of participants stratified into; morning-, neither- and evening-type individuals. It is hypothesised that, similar to depressed patients, late chronotypes will show increased accuracy to identify sad facial expressions.

\section{Methods}

\section{Participants}

The study was approved by the local ethics committee. A total of 315 participants were recruited via email advertisement, social media or personal contact.

\section{Procedures}

Participants completed an online survey (Qualtrics, Provo, UT) including a facial expression recognition task (outlined below) and three questionnaires: The Pittsburgh Sleep Quality index [PSQI] (Buysse et al., 1989); The Patient Health Questionnaire [PHQ-4] (Löwe et al., 2010) - an ultra-brief (4 questions) screening tool for anxiety and depression; and reduced Morningness-Eveningness Questionnaire [rMEQ] (Adan \& Almirall, 1991). Participants with an rMEQ score of $18+$ were determined to be morning-types (MT), $12-17$ were considered neither-types (NT) and $\leq 11$ were deemed evening-types (ET). 
The facial expression recognition task featured two basic emotions (happiness and sadness) taken from four individual characters included in the NimStim series of facial expression (www.macbrain.org/resources.htm). Images were presented in greyscale and were morphed between each prototypical and neutral expression in $10 \%$ steps $(0 \%=$ neutral, $100 \%$ $=$ full emotion, examples can be seen in Figure 1). Four examples of each emotion at each intensity were presented (two emotions $\mathrm{x}$ ten intensities $\mathrm{x}$ four examples $=80$ stimuli). Each face was also presented in a neutral expression $(0 \%=$ neutral expression, four stimuli $)$, giving a total of 84 stimuli presentations. Stimuli were presented in random order and participants indicated their responses by selecting either SAD, NEUTRAL or HAPPY on the screen. The response rule was counterbalanced across stimuli. The number of stimuli accurately classified as each emotion was recorded.

\section{Statistical treatment}

One-Way Analysis of Variance (ANOVA) and Pearson's chi-square test for independence were used to explore participant demographics and trait characteristics. For the facial expression recognition task analyses were conducted using mixed-model two-way ANOVA with chronotype (MT, NT and ET) as the between-subjects factor and facial expression (happy, sad) as the within subjects factor.

\section{Results}

A total of 315 participants completed the online study but 89 were excluded for reporting one or more co-variables; a diagnosis of depression (79), sleep disorder (15), PHQ_4 score > 9 (19) or under the age of 18 (2). Hence, a total of 226 participants were included in the final analyses. Demographic details of the study population according to chronotype are shown in Table 1. Chronotype groups were similar in terms of sleep quality (F 
$<1)$, depression and anxiety $(F(2,223)=2.92, \mathrm{p}>.05)$. Morning-types were older than both NT and ET groups $(F(2,223)=9.60, \mathrm{p}<.05)$ and a greater number of individuals in the NT group were female $\left(\chi^{2}(2,226), p<.05\right)-84$ females compared to 30 males (Table 1$)$.

\section{Table 1 near here please}

We observed a significant group $\mathrm{x}$ emotion interaction $(F(2,223)=13.05, \mathrm{P}<0.001$, $\left.n_{p}^{2}=.105\right)$. Simple effects analyses for each emotion indicated that the means for the three chronotype groups differed for sad faces $\left(F(2,223)=6.17, p=.002, n_{p}^{2}=.052\right)$ and showed a strong, but non-significant trend for happy faces $\left(F(2,223)=3.00, \mathrm{p}=.052, n_{p}^{2}=.026\right)$. Post hoc analyses, with Bonferroni correction, revealed that evening-types recognised significantly more sad faces $(M=66.90, S D=13.71)$ than either morning-types $(M=58.94$, $\mathrm{SD}=13.48, p=.004)$ or neither-types $(\mathrm{M}=61.47, \mathrm{SD}=11.73, p=.018)$ (Figure 1).

\section{Figure 1 near here please}

\section{Discussion}

We observed increased recognition of sad facial expressions in never-depressed evening-type individuals compared to morning- and neither-types. These differences were present despite groups being similar in terms of sleep quality and depression/anxiety levels, and were not driven by small differences in age and gender between groups.

Converging evidence has associated eveningness with an increased prevalence of depression (Hidalgo et al., 2009; Merikanto et al., 2013, 2015). The results of the current 
study show that healthy, never-depressed individuals display emotional biases similar to those reported in acutely depressed (Bradley et al., 1995; Peckham et al., 2010) and remitted depressed patients (Anderson et al., 2011). Cognitive theories of depression posit negative emotional biases are important in the aetiology and maintenance of depression (Beck et al., 1979). Our data, therefore, suggest that certain negative biases may exist prior to the onset of depression in evening-type individuals which may reflect a trait vulnerability marker for this disorder.

Merikanto and colleagues (2015) have suggested that ET may be at increased risk of circadian misalignment due to a clash between their circadian rhythm and social constraints which may explain the increased prevalence of health-related issues associated with eveningness including depression. If so, it might be predicted that there would be a positive association between symptom severity and the degree of social jet-lag experienced by individuals. In relation to depression, the current data and our earlier findings (Berdynaj et al., 2016) suggest that strategies such as cognitive bias modification training may be of utility in preventing the onset of depression. Alternatively, it may be beneficial for evening-types to adapt their activity schedule to match their delayed circadian clock, thereby reducing the 'clash' (Merikanto et al., 2015).

A limitation of the current study was the determination of chronotype using a single, self-report metric [the Reduced Morningness-Eveningness Questionnaire (Adan \& Almirall, 1991). In addition, the anonymous online survey required that current, previous and family history for depression and diagnosed sleep disorder was determined by self-report rather than reference to medical history or structured clinical interview. Although this method allowed us to obtain a large sample size, we cannot fully guarantee all participants met the inclusion criteria. Finally, the study was limited to measuring only two emotions; happiness and sadness. Future studies including a range of emotions are warranted. 
In conclusion, a clear association was found between ET and increased processing of negative emotional facial expressions. These findings suggest late chronotypes have emotional processing biases similar to those seen in depressed individuals and may explain why this group are more at risk of developing the disorder. This has important theoretical and clinical implications for the prevention and treatment of depression. The causes of the elevated incidence of health-related issues in evening-types as compared to morning- and neither-types is likely multifactorial and there is a need for future longitudinal studies to determine effective strategies to promote well-being in these individuals.

\section{Disclosure statement}

The authors do not have any financial interest or benefit arising from the direct application of this research. 


\section{References}

Adan A, Almirall H. 1991. Horne and Ostberg Morningness Eveningness Questionnaire - a reduced scale. Pers Individ Dif, 12: 241-53.

Anderson IM, Shippen C, Juhasz GG, Chase D, Thomas E, Downey D, Deakin JFW. 2011. State-dependent alteration in face emotion recognition in depression. Br J Psychiatry, 198: 302-08.

Beck AT. 1989. Cognitive therapy for depression and panic disorder. West J Med, 151: 31111.

Berdynaj D, Boudissa SN, Grieg MS, Hope C, Mahamed SH, Norbury R. 2016. Effect of chronotype on emotional processing and risk taking. Chronobiol Int, 33: 406-18.

Bradley BP, Mogg K, Millar N, White J. 1995. Selective processing of negative information effects of clinical anxiety, concurrent depression, and awareness. J Abnorm Psychol, 104: 532-36.

Buysse DJ, Reynolds CF, Monk TH, Berman SR, Kupfer DJ. 1989. The Pittsburgh Sleep Quality Index - a new instrument for psychiatric practice and research. Psychiatry Res, 28: 193-213.

Chan SWY, Goodwin GM, Harmer C J. 2007. Highly neurotic never-depressed students have negative biases in information processing. Psychol Med, 37: 1281-91.

Hidalgo MP, Caumo W, Posser M, Coccaro SB, Camozzato AL, Chaves MLF. 2009.

Relationship between depressive mood and chronotype in healthy subjects. Psychiatry Clin Neurosci, 63: 283-90.

Löwe B, Wahl I, Rose M, Spitzer C, Glaesmer H, Wingenfeld K, Brähler E. 2010. A 4-item measure of depression and anxiety: Validation and standardization of the Patient Health Questionnaire-4 (PHQ-4) in the general population. J Affect Disord, 122: 8695.

Merikanto I, Kronholm E, Peltonen M, Laatikainen T, Vartiainen E, Partonen T. 2015. Circadian preference links to depression in general adult population. J Affect Disord, 188: $143-48$.

Merikanto I, Lahti T, Kronholm E, Peltonen, M, Laatikainen T, Vartiainen E, Partonen T. 2013. Evening types are prone to depression. Chronobiol Int, 30: 719-25.

Peckham AD, Mchugh RK, Otto MW. 2010. A meta-analysis of the magnitude of biased attention in depression. Depress Anxiety, 27: 1135-42. 
Simor P, Zavecz Z, Palosi V, Torok C, Koteles F. 2015. The influence of sleep complaints on the association between chronotype and negative emotionality in young adults.

Chronobiol Int, 32: 1-10. 
Figure 1. Sad expression intensity-accuracy response curves (shaded areas shows standard error) for morning-type (MT), neither-type (NT) and evening-type (ET) groups. With the exception of the extreme points of the curve, ET response accuracy appears to be shifted to the left (relative to MT and NT) indicating this chronotype group are more accurate at recognising sad facial expressions. Mean response accuracy for all three groups are displayed on the right-hand side of the figure where error bars represent standard error of the mean. $(*=$ $\mathrm{p}<0.05, * *=\mathrm{p}<0.01)$.

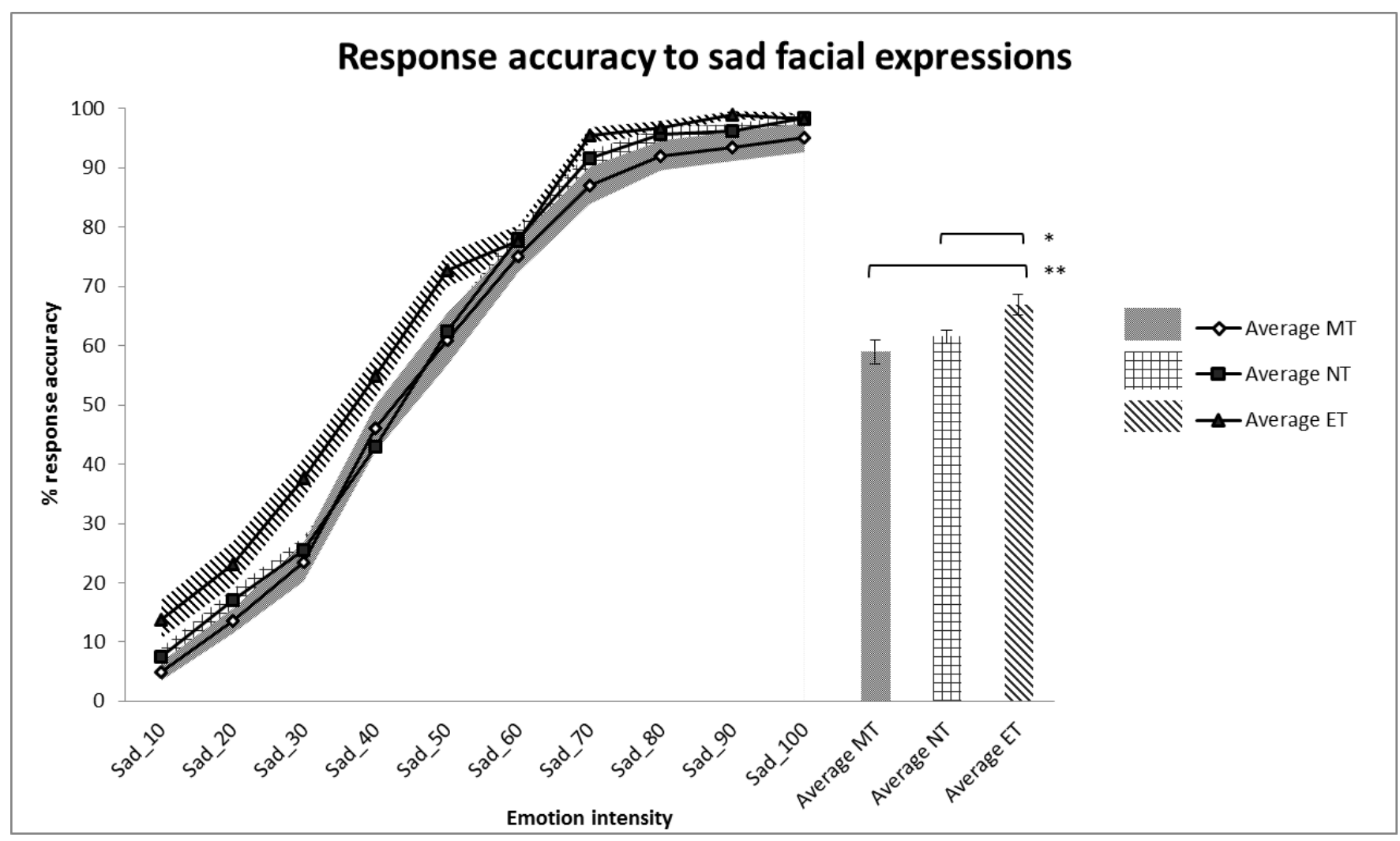


Table 1. Sample characteristics according to chronotype. Data (except Gender) are mean (SD). PSQI - Pittsburgh Sleep Quality Index; PHQ _4 - Patient Health Questionnaire.

\begin{tabular}{lccc}
\hline & \multicolumn{3}{c}{ Chronotype } \\
\hline & $\begin{array}{c}\text { Morning type } \\
(n=45)\end{array}$ & $\begin{array}{c}\text { Neither type } \\
(n=114)\end{array}$ & $\begin{array}{c}\text { Evening type } \\
(n=67)\end{array}$ \\
\hline Age & $36.93(16.92)$ & $27.71(11.97)$ & $27.28(11.11)$ \\
Gender (M/F) & $21 / 24$ & $30 / 84$ & $25 / 42$ \\
PHQ_4 & $1.44(2.03)$ & $2.37(2.20)$ & $2.16(2.23)$ \\
PSQI & $4.84(3.10)$ & $5.48(3.11)$ & $5.42(2.67)$ \\
\hline
\end{tabular}

